\title{
Minority carrier distribution in the front and base regions of a p/n GaAs-Si heterojunction solar cell and its spectral response
}

\author{
Sayantan Biswas \\ Research Scholar, Department of Physics, University of Kalyani, Kalyani-741235, West Bengal, India. \\ Amitabha Sinha \\ Professor, Department of Physics, University of Kalyani, Kalyani-741235, West Bengal, India.
}

\begin{abstract}
An analytical work has been carried out on the $\mathrm{p} / \mathrm{n} \mathrm{GaAs}-\mathrm{Si}$ heterojunction solar cell, based on the mathematical derivation presented by other researchers for the $n / p$ heterojunction solar cell. The minority carrier concentration and photocurrent contribution from both the top layer and the base layer of the cell have been studied, taking into account the effects of various parameters such as front and back surface recombination velocities, wavelength of incident light and corresponding absorption coefficient of the material. It is observed from these theoretical studies that the surface recombination velocity has a significant influence on the photocurrent contribution of the heterojunction solar cells. This suggests the role of a back-surface-field (BSF) in such devices, which may be incorporated to improve the performance of these cells.
\end{abstract}

Keywords: GaAs-Si heterojuction solar cell; minority carrier concentration; spectral response; surface recombination velocity.

\section{Introduction}

The junction formed by two semiconductors of different band gaps is known as a heterojunction, which has been widely used for making solar cells. A theoretical study on the conversion efficiency of heterojunction solar energy converters was reported by Sreedhar et al. [1]. They calculated the efficiencies of various heterojunctions, including $\mathrm{Si}-\mathrm{GaAs}$ and $\mathrm{Si}-\mathrm{GaP}$, in which they considered the band gap of the material of the first layer ( $\mathrm{Si}$ ) less than the band gap of the material of the second region (GaAs). However, it is advantageous to have the first region of larger band gap material $E_{\mathrm{g} 1}$ as compared to the band gap of the material of the second region $\mathrm{E}_{\mathrm{g} 2}$. Light of energy greater than $\mathrm{E}_{\mathrm{g} 2}$ but less than $\mathrm{Eg}_{1}$ will then pass through the first region and get absorbed by the second region. Light of energy greater than $\mathrm{E}_{\mathrm{g} 1}$ will be absorbed in the first region [2]. These absorbed photons give rise to electron-hole pairs which are separated by the field in the depletion region of the heterojunction giving rise to photocurrent. Sahai and Milnes [3] presented the analysis of such types of solar cells having larger band gap material as the first region. Their studies included the calculation of conversion efficiency of n/p GaP-Si, GaAs-Ge and other such materials. Various heterojunction pairs have been studied in detail by Milnes and Feucht [4]. An overview of transport equations for highly doped devices and heterostructures is given by Marshak [5] in detail and a review of models for heterojunction band offsets has been presented by Unlu and Nussbaum [6]. An analysis of the photovoltage of a heterojunction solar cell has been published recently [7]. Amorphous/crystalline silicon heterojunction solar cell fabrication and characterization has been discussed by Korte et al. [8]. Amorphous/crystalline heterojunction solar cells on the n-type substrates, textured in iso-propanol (IPA)-free solution, with conversion efficiencies exceeding $20 \%$ are reported by J. Kegel et al. [9]. Thus lot of research work, both experimental and theoretical, has been done on heterojunction solar cells so far. In our present paper, analytical research work has been carried out on the p/n GaAs-Si heterojunction solar cells, based on the mathematical approach discussed by Hovel [2] for a $\mathrm{n} / \mathrm{p}$ heterojunction solar cell. Since the study of minority carrier profile is helpful in understanding the physics of the device, minority carrier distributions in both the front and the base regions of the solar cell have been studied along with the photocurrent contributions from each region.

\section{Analysis}

The schematic diagram of the $\mathrm{p} / \mathrm{n}$ GaAs-Si heterojunction solar cell considered in this paper and the coordinates assumed for the purpose of analysis are shown in Figure 1. The energy band diagram of such solar cell is shown in Figure 2 [4].

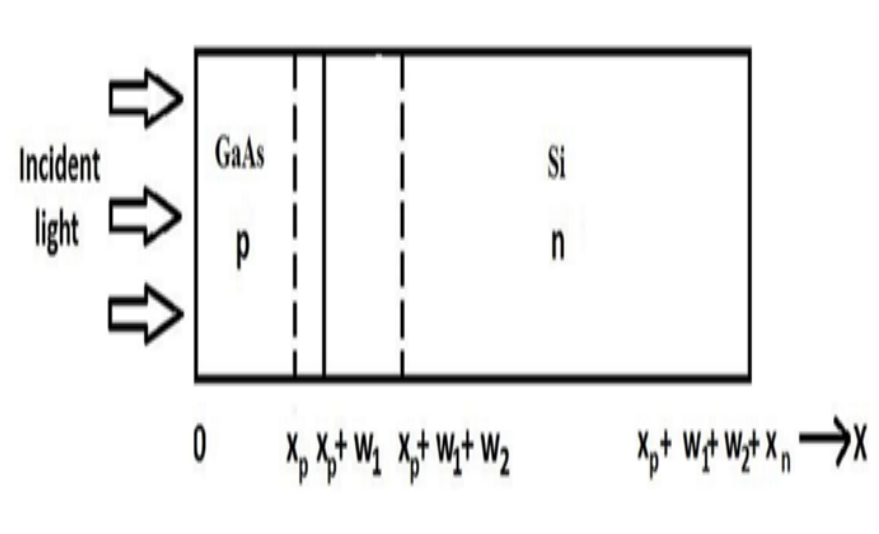

Figure 1: Schematic diagram of a $\mathrm{p} / \mathrm{n}$ heterojunction solar cell. 


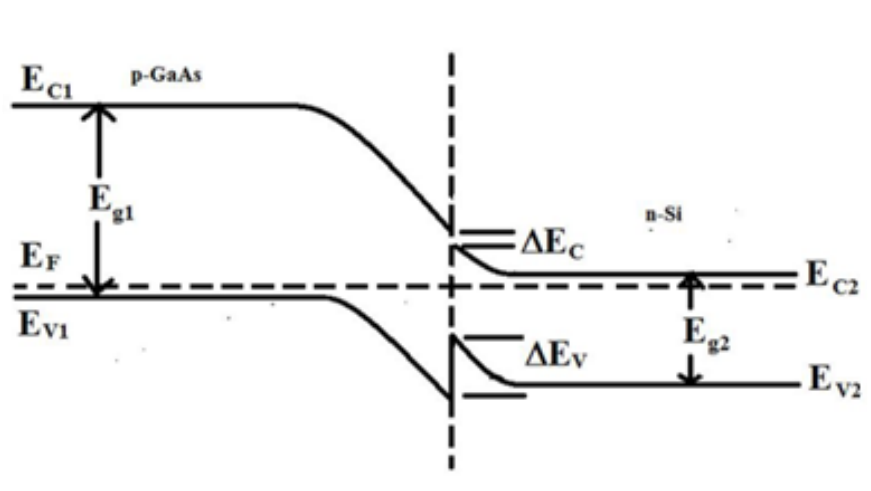

Figure 2: Energy band diagram of a p/n heterojunction solar cell

The mathematical derivation of the expression of photocurrent of an $\mathrm{n} / \mathrm{p}$ heterojunction solar cell has been given by Hovel [2]. The same approach has been used here to obtain the analytical expressions for the excess minority carrier concentration and the photocurrent contribution from the top and the base regions of a p/n GaAs-Si heterojunction solar cell.

The minority carrier continuity equations for holes and electrons are

$\frac{1}{q} \frac{d J_{p}}{d x}-G_{p}+\frac{\Delta p_{n}}{\tau_{p}}=0$

and

$\frac{1}{q} \frac{d J_{n}}{d x}+G_{n}-\frac{\Delta n_{p}}{\tau_{n}}=0$

Where $G_{p}$ and $G_{n}$ are the generation rates of electron-hole pairs in the top and base regions of the cell and given by [2] $G_{p}=\alpha_{1}(\lambda) F(\lambda)[1-R(\lambda)] \exp \left[-\alpha_{1}(\lambda) x\right]$

and

$G_{n}=\alpha_{2}(\lambda) F(\lambda)[1-R(\lambda)] \exp \left[-\alpha_{2}(\lambda) x\right]$

where $\alpha_{1}$ and $\alpha_{2}$ are the absorption coefficients of material-1 and material-2 of the cell respectively.

The boundary conditions to be employed in the front region of the cell are

at $\mathrm{x}=0 D_{n} \frac{d\left(\Delta n_{p}\right)}{d x}=S_{n} \Delta n_{p}$

at $\mathrm{x}=\mathrm{x}_{\mathrm{p}} \Delta n_{p}=0$

Using these boundary conditions, an expression for the electron concentration in the top layer may be written as

$\Delta n_{p}=\left[\frac{\alpha_{1} F(1-R) \tau_{n}}{\left(\alpha_{1}^{2} L_{n}^{2}-1\right)}\right] \times$

$\left[\frac{\left(\frac{S_{n} L_{n}}{D_{n}}+\alpha_{1} L_{n}\right) \sinh \left(\frac{x_{p}-x}{L_{n}}\right)+\exp \left(-\alpha_{1} x_{p}\right)\left\{\frac{S_{n} L_{n}}{D_{n}} \sinh \left(\frac{x}{L_{n}}\right)+\cosh \left(\frac{x}{L_{n}}\right)\right\}}{\frac{S_{n} L_{n}}{D_{n}} \sinh \left(\frac{x_{p}}{L_{n}}\right)+\cosh \left(\frac{x_{p}}{L_{n}}\right)}-\exp \left(-\alpha_{1} x\right)\right]$

Therefore the photocurrent density of the top region is

$J_{n}=-\left[\frac{q F(1-R) \alpha_{1} L_{n}}{\left(\alpha_{1}^{2} L_{n}^{2}-1\right)}\right] \times$

$$
\left[\frac{\left(\frac{S_{n} L_{n}}{D_{n}}+\alpha_{1} L_{n}\right)-\exp \left(-\alpha_{1} x_{p}\right)\left\{\frac{S_{n} L_{n}}{D_{n}} \cosh \left(\frac{x_{p}}{L_{n}}\right)+\sinh \left(\frac{x_{p}}{L_{n}}\right)\right\}}{\frac{S_{n} L_{n}}{D_{n}} \sinh \left(\frac{x_{p}}{L_{n}}\right)+\cosh \left(\frac{x_{p}}{L_{n}}\right)}-\alpha_{1} L_{n} \exp \left(-\alpha_{1} x_{p}\right)\right]
$$

Thus the spectral response of the top region is given by

$$
[S R]_{\text {top }}=\left|\frac{J_{n}}{q F(1-R)}\right|
$$

Similarly combining equations (1) and (3) with the current density equation, the hole concentration in the base layer may be obtained as

$$
\begin{aligned}
& \Delta p_{n}=\left[\frac{\alpha_{2} F(1-R) \tau_{p} \exp \left(-\alpha_{2} w_{2}\right) \exp \left(-\alpha_{1}\left(x_{p}+w_{1}\right)\right)}{\left(\alpha_{2}^{2} L_{p}^{2}-1\right)}\right] \times \\
& {\left[\cosh \left(\frac{x-x_{p}-w_{1}-w_{2}}{L_{p}}\right)-\exp \left\{-\alpha_{2}\left(x-x_{p}-w_{1}-w_{2}\right)\right\}-\right.} \\
& \left.\frac{\left[\frac{S_{p} L_{p}}{D_{p}}\left\{\cosh \left(\frac{x_{n}}{L_{p}}\right)-\exp \left(-\alpha_{2} x_{n}\right)\right\}+\sinh \left(\frac{x_{n}}{L_{p}}\right)+\alpha_{2} \exp \left(-\alpha_{2} x_{n}\right)\right] \sinh \left(\frac{x-x_{p}-w_{1}-w_{2}}{L_{p}}\right)}{\frac{S_{p} L_{p}}{D_{p}} \sinh \left(\frac{x_{n}}{L_{p}}\right)+\cosh \left(\frac{x_{n}}{L_{p}}\right)}\right]
\end{aligned}
$$

Where the following boundary conditions have been used for the base layer of the cell

$$
\begin{aligned}
& \text { at } x=x_{p}+w_{1}+w_{2} \Delta p_{n}=0 \\
& \text { at } x=x_{p}+w_{1}+w_{2}+x_{n} D_{p} \frac{d \Delta p_{n}}{d x}=s_{p} \Delta p_{n}
\end{aligned}
$$

The corresponding photocurrent density for the base region is given by

$$
\begin{aligned}
& J_{p}=-\left[\frac{\alpha_{2} F(1-R) L_{p} \exp \left(-\alpha_{2} w_{2}\right) \exp \left(-\alpha_{1}\left(x_{p}+w_{1}\right)\right)}{\left(\alpha_{2}^{2} L_{p}^{2}-1\right)}\right] \times \\
& {\left[\alpha_{2} L_{p}-\frac{\frac{S_{p} L_{p}}{D_{p}}\left\{\cosh \left(\frac{x_{n}}{L_{p}}\right)-\exp \left(-\alpha_{2} x_{n}\right)\right\}+\sinh \left(\frac{x_{n}}{L_{p}}\right)+\alpha_{2} L_{p} \exp \left(-\alpha_{2} x_{n}\right)}{\frac{S_{p} L_{p}}{D_{p}} \sinh \left(\frac{x_{n}}{L_{p}}\right)+\cosh \left(\frac{x_{n}}{L_{p}}\right)}\right]}
\end{aligned}
$$

Hence the spectral response of the base region is given by

$[S R]_{\text {base }}=\left|\frac{J_{p}}{q F(1-R)}\right|$

The depletion widths $\mathrm{w}_{1}$ and $\mathrm{w}_{2}$ are determined by following the published literature [2].

The analysis presented here is exactly similar to the analysis for a GaAs-Si $\mathrm{n} / \mathrm{p}$ heterojunction solar cell published earlier $[2,11]$.

\section{Results and Discussions}

Calculations were performed using the mathematical expressions derived in the preceding section and the results are shown in the figures presented here. The concentration of acceptor atoms taken in the calculations for the top layer is $1.4 \times 10^{18} \mathrm{~cm}^{-3}$. The values of diffusion length and diffusion coefficient for electrons in the top layer are taken as $2 \mu \mathrm{m}$ and $200 \mathrm{~cm}^{2} \mathrm{~s}^{-1}$ respectively. The concentration of donor atoms taken for the base region is $10^{15} \mathrm{~cm}^{-3}$. The values of diffusion length and diffusion coefficient for holes in the base region are taken as $654.8 \mu \mathrm{m}$ and $12.4 \mathrm{~cm}^{2} \mathrm{~s}^{-1}$ following the relationship given by J. G. Fossum [10]. The width of the top 
and base region of the cell is taken as $0.6 \mu \mathrm{m}$ and $100 \mu \mathrm{m}$ respectively.

Using equation (7), the excess minority carrier concentration in the p-type top layer of the $\mathrm{p} / \mathrm{n}$ GaAs-Si heterojunction solar cell as a function of position for various values of front surface recombination velocity $S_{n}$ has been plotted in Figure 3. It is observed from the figure that as the front surface recombination velocity is increased excess minority carrier electron concentration is decreased. This is due to the reason that as a result of recombination at the front surface, many minority carriers are lost there resulting in a fall in $\Delta n_{p}$ for larger values of $S_{n}$.

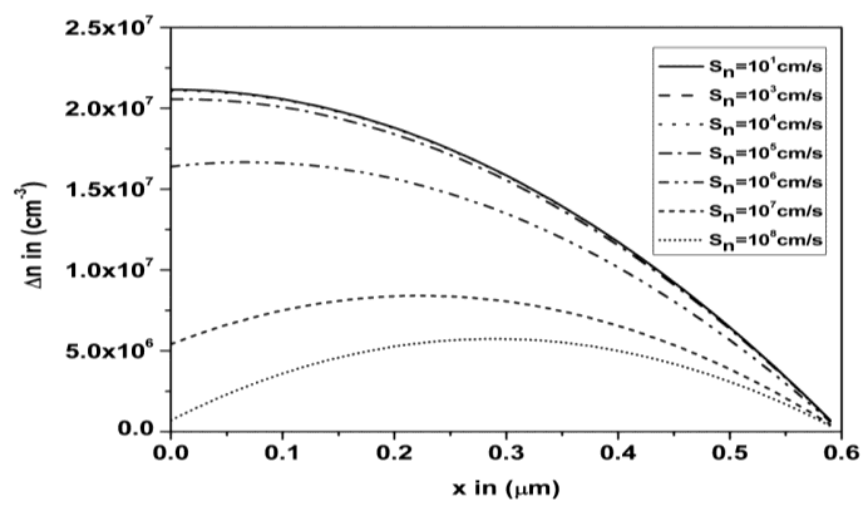

Figure 3: Variation of excess minority carrier concentration with position corresponding to different values of front surface recombination velocity $S_{n}$.

Figure 4 shows the variation of excess minority carrier concentration with position for various values of absorption coefficient $\alpha$. It is found that the excess minority carrier electron concentration rises with the increase of the value of absorption coefficient $\alpha$. As most of the wavelengths corresponding to higher values of $\alpha$ are absorbed in the front region, the excess carrier concentration for these absorbed photons increases in this region.

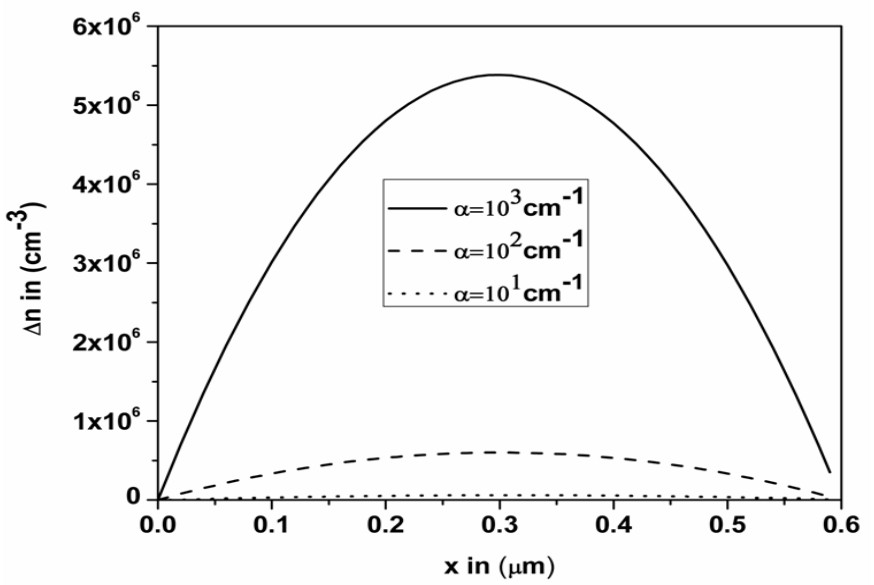

Figure 4: Variation of excess minority carrier concentration with position corresponding to different values of absorption coefficient $\alpha$.
Figure 5 shows the variation of spectral response with wavelength of the top layer for various values of front surface recombination velocity $S_{n}$. As seen from the figure, spectral response is large for smaller values of $S_{n}$. This is because of the fact that for smaller values of $S_{n}$, the loss of minority carriers due to recombination at the front surface is small, and hence the spectral response improves.

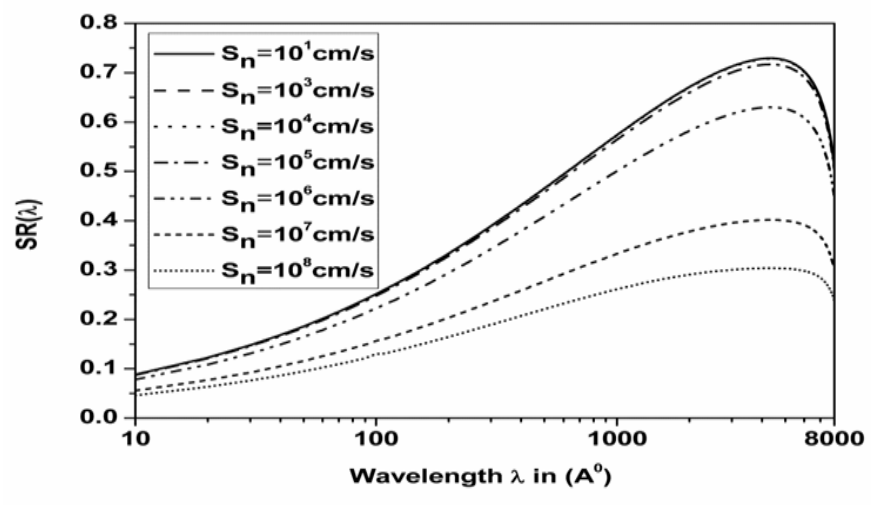

Figure 5: Variation of spectral response of the top region with wavelength $\lambda$ corresponding to different values of front surface recombination velocity $S_{n}$.

Using equation (10), the variation of excess minority carrier hole concentration in the base region, as a function of position corresponding to different values of back surface recombination velocity $S_{p}$, has been plotted in Figure 6. From the figure it is observed that the minority carrier concentration is much higher near the back surface, for smaller values of back surface recombination velocity. This is explained from the fact that low recombination at the rear leads to large accumulation of the carriers there.

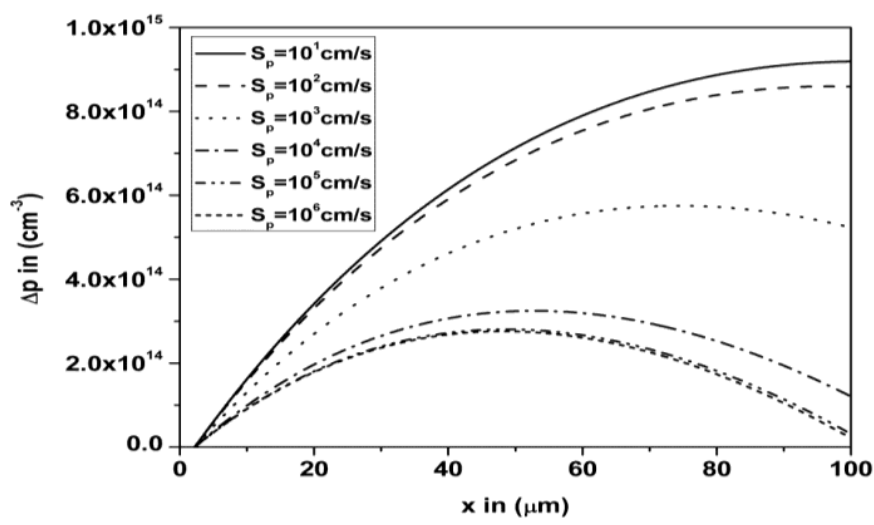

Figure 6: Variation of excess minority carrier concentration with position corresponding to different values of back surface recombination velocity $S_{p}$

Figure 7 represents the variation of excess minority carrier hole concentration as a function of position for various values of absorption coefficient $\alpha$. 


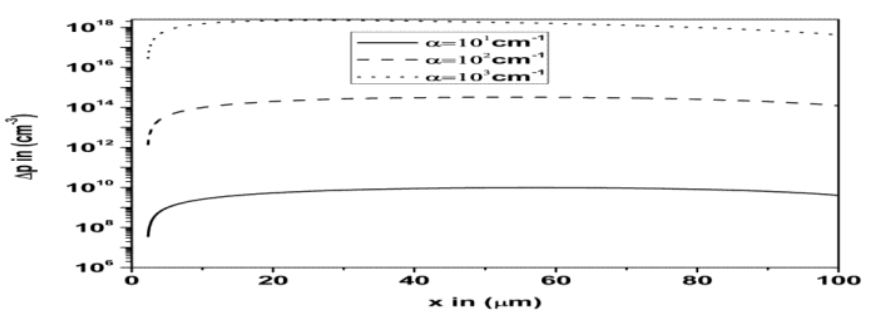

Figure 7: Variation of excess minority carrier concentration with position corresponding to different values of absorption coefficient $\alpha$.

The variation of spectral response contribution from the base layer of the cell as a function of wavelength $\lambda$ corresponding to different values of back surface recombination velocity $S_{p}$ is shown in Figure 8. From the figure it is observed that the spectral response of the base region depends on the surface recombination velocity significantly.

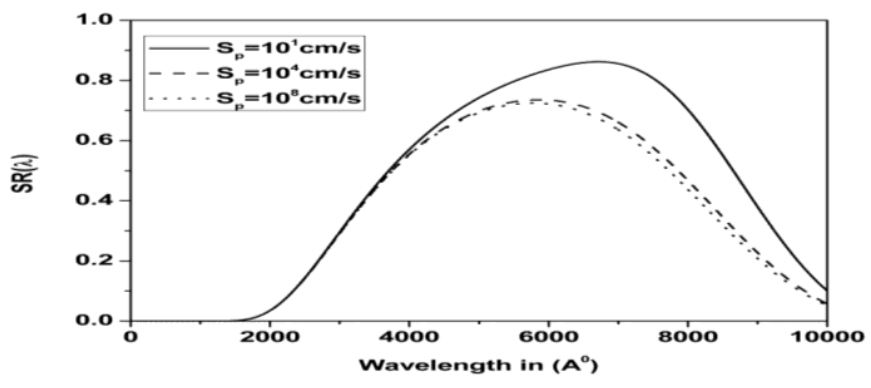

Figure 8. Variation of spectral response with wavelength $\lambda$ corresponding to different values of surface recombination velocity $S_{\mathrm{p}}$.

Figure 9 shows the total spectral response of the entire cell, consisting of the front region and the base region. From the figure it is observed that the spectral response attains maximum for the wavelength about $6000 \AA$.

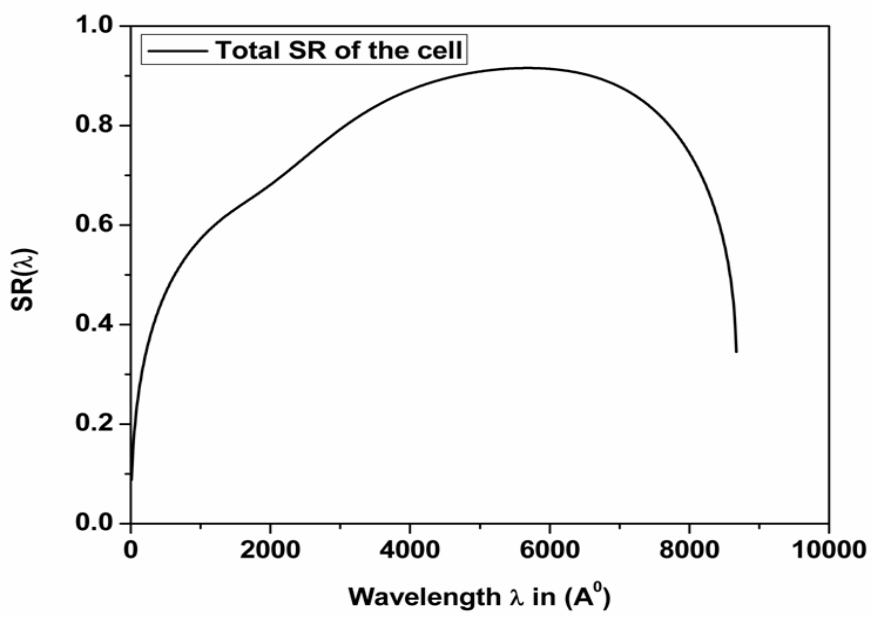

Figure 9: Total spectral response of the entire solar cell.
It may be mentioned here that the results obtained in this paper are similar to those obtained earlier for a GaAs-Si n/p heterojunction solar cell [11]. Hence we again suggest a heterojunction solar cell structure as shown in Figure 10, which may yield improved photocurrent.

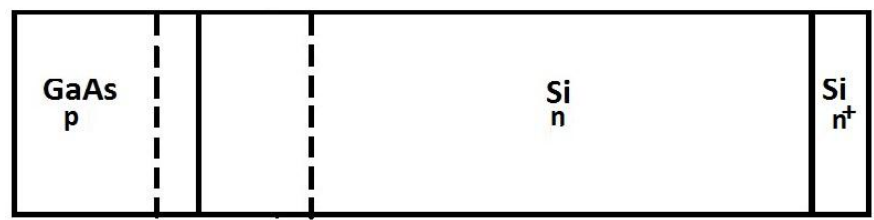

Figure 10: Suggested schematic diagram of a $\mathrm{p} / \mathrm{n} \mathrm{GaAs}-\mathrm{Si}$ heterojunction solar cell with a back-surface-field.

\section{Conclusion}

The excess minority carrier concentrations and photocurrent contributions from the top layer and from the base layer of a $\mathrm{p} / \mathrm{n}$ GaAs-Si heterojunction solar cell have been studied analytically in this paper. It is found that the excess minority carrier concentrations and photocurrent contributions of the top region and base region depend significantly on the absorption coefficient and as well as on the surface recombination velocities. The results are consistent with those obtained for an $\mathrm{n} / \mathrm{p}$ GaAs-Si heterojunction solar cell. A structure with a low-high $\mathrm{nn}^{+}$junction at the back of the normal cell is suggested to give improved performance.

\section{Acknowledgements}

We are grateful to the Department of Science and Technology, Govt. of India, for financial support under the DST-PURSE Programme, granted to the University of Kalyani. We thank the authorities of Indian Association for the Cultivation of Science, Kolkata, India, for allowing us to consult their library.

\section{References}

[1] Sreedhar, A.K., Sharma, B.L., and Purohit, R.K., 1969, "Efficiency calculations of heterojunction solar energy converters," IEEE Transaction Electron Devices, 16, pp. 309-312.

[2] Hovel, H.J., 1975, "Semiconductors and Semimetals: solar cells," 11, Academic Press, New York, pp. 127139.

[3] Sahai, R., and Milnes, A.G., 1970, "Heterojunction solar cell calculations," Solid State Electronics, 13, pp. 1289-1299.

[4] Milnes, A.G., and Feucht, D.L., 1972 "Heterojunction and Metal-Semiconductor Junctions," Academic Press, New York.

[5] Marshak, A.H., 1987, "Transport equations for highly doped devices and heterostructures," Solid State Electronics, 30, pp. 1089-1093. 
International Journal of Applied Engineering Research ISSN 0973-4562 Volume 11, Number 6 (2016) pp. $4136-4140$

(C) Research India Publications. https://dx.doi.org/10.37622/IJAER/11.6.2016.4136-4140

[6] Unlu, H., and Nussbaum, A., 1987, "A review of models for heterojunction band offsets," Solid State Electronics, 30, pp. 1095-1098.

[7] Chao, X., He, Y.R., and Wei, G.K., 2011, "Photovoltage analysis of a heterojunction solar cell," Chin Phys. B, 20, pp. 057302.

[8] Korte, L., Conrad, E., Angermann, H., Stangl, R., and Schmidt, M., 2009, "Advances in a-Si: H/c-Si heterojunction solar cell fabrication and characterization," Solar Energy Materials and Solar Cells, 93, pp. 905-910.

[9] Kegel, J., Angermann, H., Sturzebecher, U., Conrad, E., Mews, M., Korte, L., and Stegemann, B., 2014, "Over 20\% conversion efficiency on silicon heterojunction solar cells by IPA-free substrate texturization," Applied Surface Science, 301, pp. 5662.

[10] Fossum, J. G., 1976, “Computer-Aided Numerical Analysis of Silicon Solar Cells," Solid State Electronics, 19, pp. 269-277.

[11] Biswas, S., Biswas, A. K., Chatterjee, A., and Sinha, A., 2016, "An analytical study of a GaAs-Si n/p heterojunction solar cell and suggestion for a structure for improved performance," International Journal of Applied Engineering Research, 11, pp. 1855-1858. 